\title{
Expected Radial Column Density of Methylcyanopolyynes in CW Leonis (IRC+10216)
}

\author{
Raul G. E. Morales ${ }^{1}$, Carlos Hernández ${ }^{2}$ \\ ${ }^{1}$ Centre for Environmental Sciences and Department of Chemistry Faculty of Sciences, Universidad de \\ Chile, Santiago, Chile \\ ${ }^{2}$ Department of Chemistry, Faculty of Basic Sciences Universidad Metropolitana de Ciencias de la \\ Educación, Santiago, Chile \\ Email: correo@raulmorales.cl, carloshernandez.carlos@gmail.com
}

Received 31 January 2015; accepted 24 August 2015; published 27 August 2015

Copyright (C) 2015 by authors and Scientific Research Publishing Inc.

This work is licensed under the Creative Commons Attribution International License (CC BY).

http://creativecommons.org/licenses/by/4.0/

(c) (i) Open Access

\begin{abstract}
Methylcyanopolyynes $\left(\mathrm{CH}_{3}-[\mathrm{C} \equiv \mathrm{C}]_{n}-\mathrm{CN}\right)$ are a particular kind of linear molecular wires, where the first three oligomers have been detected in the interstellar medium, particularly in CW Leonis (IRC + 10216), as well as in the envelopes of carbon-rich stars in a similar way to the unsubstituted cyanopolyynes. Based on the projected natural distribution in cold clouds under LTE, we have determined the radial column density of new expected methylcyanopolyynes to be present in CW Leonis (IRC + 10216). By following, we have made use of the inner molecular resistances of the internal charge transfer process presenting in these oligomeric species in order to determine the reactivity trends between them. Therefore, geometrical parameters and dipole moments determinations for these methylcyanopolyynes involving the $n=1$ to 14 molecular species were obtained from $A b$ initio molecular orbital calculations by means of a GAUSSIAN Program, using a restricted Hartree-Fock approach and 6-311G* basis set. Our results present a similar behavior observed in cyanopolyynes, where this series reaches a saturation level at the $14^{\text {th }}$ oligomer with a maximum dipole moment of $8.21 \pm 0.01$ (Debyes). Thus, this molecular wire model permits us to comprehend how these methylcyanopolyynes reach a maximum length in such chemical environment, in agreement to the astronomical observations and cosmological chemical models. The following $\mathrm{CH}_{3} \mathrm{C}_{9} \mathrm{~N}$ and $\mathrm{CH}_{3} \mathrm{C}_{11} \mathrm{~N}$ oligomers in $\mathrm{CW}$ Leonis should be expected near to $3.52 \times 10^{10}$ [ $\mathrm{cm}^{2}$ ] and $1.82 \times 10^{10}\left[\mathrm{~cm}^{2}\right]$, respectively.
\end{abstract}

\section{Keywords}

Methylcyanopolyynes, Dipole Moments, Inner Molecular Resistances, Organic Molecular Wires, ISM Radial Column Density 


\section{Introduction}

New observational evidences in some interstellar mediums (ISM) such as circumstellar shells and cold nebulae, it has been recently reported in Sagittarius B2, HL Tauri, Rho Ophiuchi cloud complex and Orion KL, between others, show the presence and abundance of different molecular species [1]-[8]. Large molecules in heterogeneous astronomical environments is one of the main goals of the frontier Astrochemistry, where project as ALMA (Atacama Large Millimeter Array) is being implemented in the south hemisphere, in order to reach new molecular observations and therefore, new explanations of complex molecular systems such as fullerenes in the ISM originated from long linear molecular compounds such as cyanopolyynes.

Several smaller members of the polyynic series have been detected in the ISM, particularly in cold circumstellar envelopes surrounding carbon-rich stars. These unsaturated carbon chains comprise at one end by hydrogen or methyl group and a $\mathrm{CN}$ group in the other extreme, a classical electron acceptor group [9]. The rotational spectra of smaller members of the simplest cyanopolyynes up to $\mathrm{HC}_{17} \mathrm{~N}$ are well known at the laboratory [10], but under hard experimental conditions and extreme detection sensitivity. However, $\mathrm{HC}_{11} \mathrm{~N}$ has been the last-discovered large linear interstellar molecule [11] [12]. Whenever spectral measurements of these types of molecular wires involving the CN group [13] [14] from various interstellar regions have been reported since 1978 up to date, the astronomical observations have systematically proved the difficult to detect molecular wires longer than five units.

These linear oligomeric molecular structures are constituted by an electron-donor group (D), in this case $\mathrm{CH}_{3}$ group, a molecular wire bridge (W), represented by the unsaturated carbon chain $\left(-[C \equiv C]_{n}-\right)$, and the $C N$ electron-acceptor group (A) [15]-[17]. By following, when these molecules are formed an electronic charge transfer process occurring from $\mathrm{D}$ to $\mathrm{A}$ through $\mathrm{W}$, i.e., materialized the dipole moment. In several previous studies [18]-[21], we have developed an one-dimensional molecular model for these systems based on the electronic conduction properties of linear conjugated oligomeric compounds of the D-W-A type. These studies have allowed us to focus our particular attention on these new interstellar cyanopolyynes.

In the present work, we have extended our cyanopolyynes study [19] to the methylcyanopolyynes, in order to find out the expected radial column density of new species to be present in CW Leonis (IRC +10216). Geometrical and dipole moment parameters calculated from $A b$ initio molecular orbital calculations, permitted us to determine the molecular resistances, necessary data to correlate the radial column densities in a similar trend previously observed in cyanopolyynes species [19].

\section{The One-Dimensional Conduction Model}

Based on our one-dimensional conduction model for D-W-A molecular systems developed some years ago [9] [18], we have determined the molecular resistance of the first fourteen methylcyanopolyynes and the linear molecular resistivity. Therefore, the dipole moment of every linear $n^{\text {th }}$ oligomer $\left(\mu_{n}\right)$ can be represented as [9]:

$$
\mu_{n}=\mu_{o}+\mu_{\infty}\left\{1-\mathrm{e}^{-\gamma L(n)}\right\}
$$

where $\mu_{0}$ is the dipole moment of the first compound of the oligomeric series without a bridge unit $(n=0), \mu_{\infty}$ is a molecular constant of the oligomeric series at the limit value for $L \rightarrow \infty, L(n)$ is the molecular wire length of the $n^{\text {th }}$ oligomer $\left(-[C \equiv C]_{n}-\right)$, and $\gamma$ is the molecular wire conduction constant. Thus, the inner resistance of the molecular wire can be depicted as follow [9]:

$$
R(L)=\left(h / 2 \mathrm{e}^{2}\right)\left[\mathrm{e}^{g L}-1\right]
$$

where $\left(h / 2 \mathrm{e}^{2}\right)=12.91 \mathrm{k} \Omega$. However, in order to separate the linear and non linear contributions of the molecular wire resistance, we can expand $R(L)$ in Equation (2) as a Maclaurin series:

$$
R(L)=12.91\left[\gamma L+1 / 2 ! \gamma^{2} L^{2}+\cdots+1 / m ! \gamma^{m} L^{m}\right]
$$

where the first term defines the linear contribution given by $R_{l}=12.91[\gamma L](\mathrm{k} \Omega)$ and the remainder terms of the series define the non linear contribution of the molecular wire resistance. Thus, the linear resistivity $(\rho)$, an intrinsic property of the conductor wires, can be calculated as $\rho=R_{\mathrm{l}} \cdot(\mathrm{S} / \mathrm{L})$, where $\mathrm{S}$ is the molecular wire cross-section estimated to be $4.5 \AA^{2}[18]$. 


\section{Results and Discussion}

The best molecular geometry optimization and the electronic charge distribution as a function of the length of the molecular wire for the methylcyanopolyynes under study were obtained by means of the GAUSSIAN Quantum Mechanics Program software [22]. From this calculation program we have used the restricted Hartree-Fock (RHF) approach and the $6-311 \mathrm{G}^{*}$ basis set, up to reach the best linear equilibrium geometry. All these oligomeric compounds, from $n=1$ to 14 , have been well described by means of these molecular-orbital calculations.

However, it is well known fact by experimentalist the inherent difficulties that these cyanopolyynes present for laboratory synthesis according to increase the length of the molecular series, due to high molecular instability, as well as, hard experimental conditions in order to reach dipole moment measurements in vacuum. Therefore, it is not possible to compare systematic dipole moment measurements to our or other calculations, however, the fresh results providing by the recent Astrochemistry research can open new expectative in a near future.

In spite of the scarce studies on this subject, our results permit to project the interstellar chemistry of these particular methylcyanopolyynes [9] [19]. Hence, $L(n)$ as the molecular wire lengths and $\mu_{n}$ as the dipole moments of these methylcyanopolyynes were calculated using this theoretical approach (Table 1). Figure 1 shows the expected interdependence between $\mu_{n}$ and $L(n)$ in agreement to the molecular model developed for these molecular wires [9]. In this Figure, the observed trend is better than $r^{2}>0.999$ and the final parameters described by Equation (1) in Figure 1 are presented in Table 2.

Furthermore, from Figure 1 we can observe how this molecular series converge to a maximum dipole moment as function of the polyynic wire in a similar behavior than the cyanopolyynes [19]. Thus, according to Equation (1), the observed limit is determined by $\left(\mu_{o}+\mu_{\infty}\right)$, where the dipole moment in this oligomeric series converge to $8.21 \pm 0.01$ (Debyes).

Therefore, the oligomeric series under study have been extended to the first fourteen species, where after the $\mathrm{CH}_{3} \mathrm{C}_{29} \mathrm{~N}$ compound, the dipole moment does not change significantly.

On the other hand, the $\gamma$ molecular wire conduction constant of methylcyanopolyynes exhibits a similar behavior to the cyanopolyynes [19]. Certainly, the methyl group induces a slight perturbation to the molecular wire nevertheless the observed change is lower than $3 \%$.

Thus, by means of this $\gamma$ conduction constant, we have determined the molecular resistances as a function of the wire length according to Equation (2). Table 3 depicts the molecular resistance of these methylcyanopolyynes and we have determined a linear resistivity of $74.9 \mu \Omega / \mathrm{cm}$, similar to cyanopolyynes $(72.6 \mathrm{k} \Omega / \mathrm{cm})$ and other molecular wires previously reported [19].

Based on these results, we have used the molecular resistance as a criterion for chemical reactions feasibility of these linear molecular species [19]. Thus, the molecular resistance to the internal charge transfer emerges as

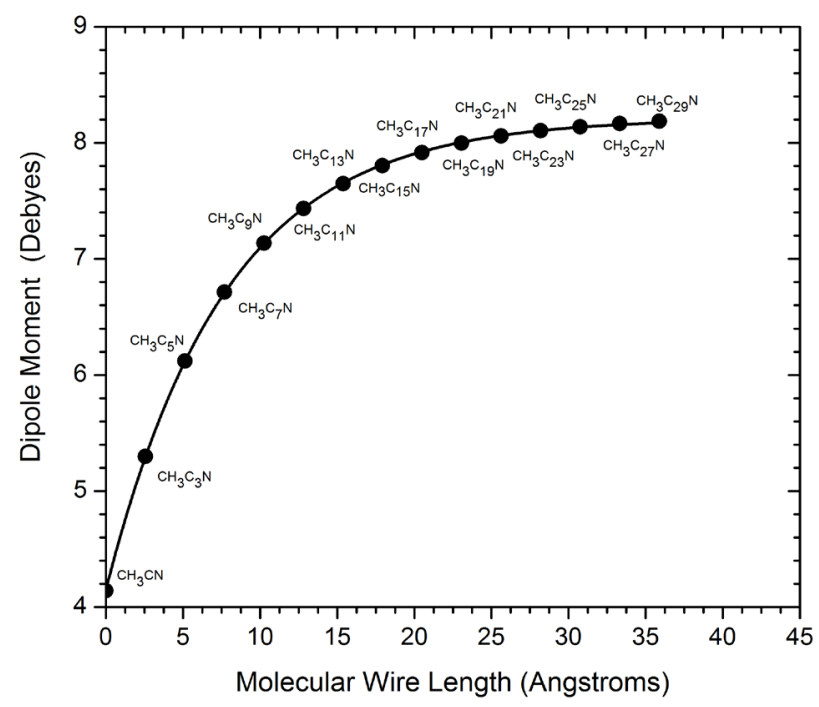

Figure 1. Dipole moments of the methylcyanopolyynes $(n=0$ to 14) versus the molecular wire lengths in the RHF approach and 6-311G* basis set. 
Table 1. Molecular wire lengths and dipole moments of the Methylcyanopolyynes according to Gaussian calculations in the RHF approach and the 6-311G* basis set.

\begin{tabular}{cccc}
\hline Molecular Series & Formula & $\begin{array}{c}\text { Molecular Wire } \\
\text { Length }(\AA)\end{array}$ & $\begin{array}{c}\text { Dipole Moment } \\
\text { (Debye) }\end{array}$ \\
$\mathrm{CH}_{3}-\mathrm{C} \equiv \mathrm{N}$ & $\mathrm{CH}_{3} \mathrm{CN}$ & 0.00 & 4.14 \\
$\mathrm{CH}_{3}-(\mathrm{C} \equiv \mathrm{C})_{1}-\mathrm{C} \equiv \mathrm{N}$ & $\mathrm{CH}_{3} \mathrm{C}_{3} \mathrm{~N}$ & 2.56 & 5.30 \\
$\mathrm{CH}_{3}-(\mathrm{C} \equiv \mathrm{C})_{2}-\mathrm{C} \equiv \mathrm{N}$ & $\mathrm{CH}_{3} \mathrm{C}_{5} \mathrm{~N}$ & 5.13 & 6.12 \\
$\mathrm{CH}_{3}-(\mathrm{C} \equiv \mathrm{C})_{3}-\mathrm{C} \equiv \mathrm{N}$ & $\mathrm{CH}_{3} \mathrm{C}_{7} \mathrm{~N}$ & 7.69 & 6.71 \\
$\mathrm{CH}_{3}-(\mathrm{C} \equiv \mathrm{C})_{4}-\mathrm{C} \equiv \mathrm{N}$ & $\mathrm{CH}_{3} \mathrm{C}_{9} \mathrm{~N}$ & 10.25 & 7.14 \\
$\mathrm{CH}_{3}-(\mathrm{C} \equiv \mathrm{C})_{5}-\mathrm{C} \equiv \mathrm{N}$ & $\mathrm{CH}_{3} \mathrm{C}_{11} \mathrm{~N}$ & 12.82 & 7.43 \\
$\mathrm{CH}_{3}-(\mathrm{C} \equiv \mathrm{C})_{6}-\mathrm{C} \equiv \mathrm{N}$ & $\mathrm{CH}_{3} \mathrm{C}_{13} \mathrm{~N}$ & 15.38 & 7.65 \\
$\mathrm{CH}_{3}-(\mathrm{C} \equiv \mathrm{C})_{7}-\mathrm{C} \equiv \mathrm{N}$ & $\mathrm{CH}_{3} \mathrm{C}_{15} \mathrm{~N}$ & 17.94 & 7.80 \\
$\mathrm{CH}_{3}-(\mathrm{C} \equiv \mathrm{C})_{8}-\mathrm{C} \equiv \mathrm{N}$ & $\mathrm{CH}_{3} \mathrm{C}_{17} \mathrm{~N}$ & 20.50 & 7.91 \\
$\mathrm{CH}_{3}-(\mathrm{C} \equiv \mathrm{C})_{9}-\mathrm{C} \equiv \mathrm{N}$ & $\mathrm{CH}_{3} \mathrm{C}_{19} \mathrm{~N}$ & 23.07 & 8.00 \\
$\mathrm{CH}_{3}-(\mathrm{C} \equiv \mathrm{C})_{10}-\mathrm{C} \equiv \mathrm{N}$ & $\mathrm{CH}_{3} \mathrm{C}_{21} \mathrm{~N}$ & 25.63 & 8.06 \\
$\mathrm{CH}_{3}-(\mathrm{C} \equiv \mathrm{C})_{11}-\mathrm{C} \equiv \mathrm{N}$ & $\mathrm{CH}_{3} \mathrm{C}_{23} \mathrm{~N}$ & 28.19 & 8.10 \\
$\mathrm{CH}_{3}-(\mathrm{C} \equiv \mathrm{C})_{12}-\mathrm{C} \equiv \mathrm{N}$ & $\mathrm{CH}_{3} \mathrm{C}_{25} \mathrm{~N}$ & 30.75 & 8.14 \\
$\mathrm{CH}_{3}-(\mathrm{C} \equiv \mathrm{C})_{13}-\mathrm{C} \equiv \mathrm{N}$ & $\mathrm{CH}_{3} \mathrm{C}_{27} \mathrm{~N}$ & 33.31 & 8.17 \\
$\mathrm{CH}_{3}-(\mathrm{C} \equiv \mathrm{C})_{14}-\mathrm{C} \equiv \mathrm{N}$ & $\mathrm{CH}_{3} \mathrm{C}_{29} \mathrm{~N}$ & 35.88 & 8.19 \\
\hline & & &
\end{tabular}

Table 2. Dipole moment parameters of the Methylcyanopolyynes.

\begin{tabular}{cc}
\hline Dipole Parameters & \\
\hline$\mu_{o}$ (Debyes) & $4.147 \pm 0.007$ \\
$\mu_{\infty}$ (Debyes) & $4.065 \pm 0.007$ \\
$\gamma\left(\AA^{-1}\right)$ & $0.1292 \pm 0.0005$ \\
\hline
\end{tabular}

Table 3. Inner molecular resistance of the Methylcyanopolyynes.

\begin{tabular}{ccc}
\hline $\mathrm{CH}_{3}-(\mathrm{C} \equiv \mathrm{C})_{\mathrm{n}}-\mathrm{C} \equiv \mathrm{N}$ & $\mathrm{R}(\mathrm{k} \Omega)$ \\
1 & 5.06 \\
2 & 12.14 \\
3 & 21.96 \\
4 & 35.63 \\
5 & 54.74 \\
6 & 81.26 \\
7 & 118.2 \\
8 & 169.6 \\
9 & 241.4 \\
10 & 341.1 \\
11 & 479.9 \\
12 & 673.1 \\
13 & 942.0 \\
14 & 1318 \\
\hline
\end{tabular}


an indicator of the polarity strength of the ground state during the molecular formation process for every one molecule of the polyynic series. Consequently, every new polyynic unit extension of the molecular wire of the methylcyanopolyynes gradually presents an additional resistance to the internal charge transfer process and, subsequently, their reaction feasibility necessarily decreases, weakening the attraction force of the CN electron-acceptor group trough the molecular wire.

Therefore it is not possible to expect long wire cyanopolyynes when its internal charge transfer activation energy is higher than the thermal energy of the reaction bulk, in other words, when the dipole moment reaches infinitesimal changes between two molecules of the series. This observation is particularly interesting to the interstellar cold dense-clouds. In this case the molecular reactivity for every molecule of the series must be understood how a dependent parameter of the sequential reaction scheme and under the same LTE conditions in a bulk delimited by low-temperature dense-cloud regions.

Therefore, the role of the $\mathrm{CN}$ group in the chemical reactions associated to the cyanopolyynes synthesis is determined by the electronic feasibility of the charge transfer between the molecular wire and the electron acceptor group. Thus, we can expect a relationship between the oligomeric species distribution and the molecular resistance to the internal charge transfer of the molecular wires that determines the final probability of the molecular array density under LTE.

IRC +10216 or CW Leonis, a carbon-rich star embedded in a thick dust envelope, has been a specific natural reactor of molecular reactions. This stellar source has been well studied both observationally and theoretically and Millar et al. [7] have developed chemical models in order to reproduce detailed radial distributions of different molecules. In particular, they report the cyanopolyynes chemistry based on the positive ion-molecule and neutral-neutral reactions leading to the production of these oligomers. Reactions including the radical $\mathrm{CN}$ and hydrocarbons were involved in the formation of cyanopolyynes, as they are in dense clouds, but reactions between the radical $\mathrm{C}_{2} \mathrm{H}$ and smaller cyanopolyynes were far more important in the IRC +10216 envelope chemistry.

Radial column densities for the cyanopolyynes in IRC +10216 are well represented by the Millar et al. model [7], which includes several cyanopolyynes and the only three observed methylcyanopolyynes. Therefore, we have made use of the radial column densities of these last reported oligomers [7]. In Figure 2, we can appreciate the methylcyanopolyynes expected behavior between radial column density and the molecular resistance. A very good linear correlation can be observed in the logarithmic scale in a similar behavior than the cyanopolyynes previously reported [19]. Therefore, this result in a new molecular series as the methylcyanopolyynes gives a new basement to our hypothesis respect to the chemical reaction feasibility in terms of the molecular wire length. Furthermore, the present methodology determines a new approximation to the radial column density estimation for astronomical observations in order to project the presence of new molecular species of these oligomeric varieties.

Thus, based on this linear correlation we have estimated the expected molecular distributions through the radial column density of this series from the relationship established by Figure 2. By following the radial column density (RCD) of these methylcyanopolyynes can be determined as follow:

$$
\log (\mathrm{RCD})=12.95-1.548 \log (R(L))
$$

where $R(L)$ is in $\mathrm{k} \Omega$ and $\mathrm{RCD}$ in $\mathrm{cm}^{2}$. By following, the expected new radial column densities for the next two members of this oligomers in CW Leonis, $\mathrm{CH}_{3} \mathrm{C}_{9} \mathrm{~N}$ and $\mathrm{CH}_{3} \mathrm{C}_{11} \mathrm{~N}$, should be expected near to $3.52 \times 10^{10}$ [ $\mathrm{cm}^{2}$ ] and $1.82 \times 10^{10}\left[\mathrm{~cm}^{2}\right]$, respectively.

\section{Conclusions}

Our results show a new route of linear oligomeric species analysis presenting in the ISM. Furthermore, the methodology developed from inner resistance to the dipole moments in these molecular wire systems opens new lines of research in the astronomical distribution and detection limits of these specific chemicals.

Particularly, in the present work, we show how dipole moments of these oligomeric series increase with chain length up to reach a certain saturation point, as can be seen in cyanopolyynes [19] and methylcyanopolyynes (this work), where $\mathrm{R}-[\mathrm{C} \equiv \mathrm{C}]_{14}-\mathrm{CN}$ establishes a molecular limit in every series. Therefore, we have developed a simple approach extendable to other similar molecular series.

Furthermore, our results and methodology on these molecular wires determine new tools of analysis to be 


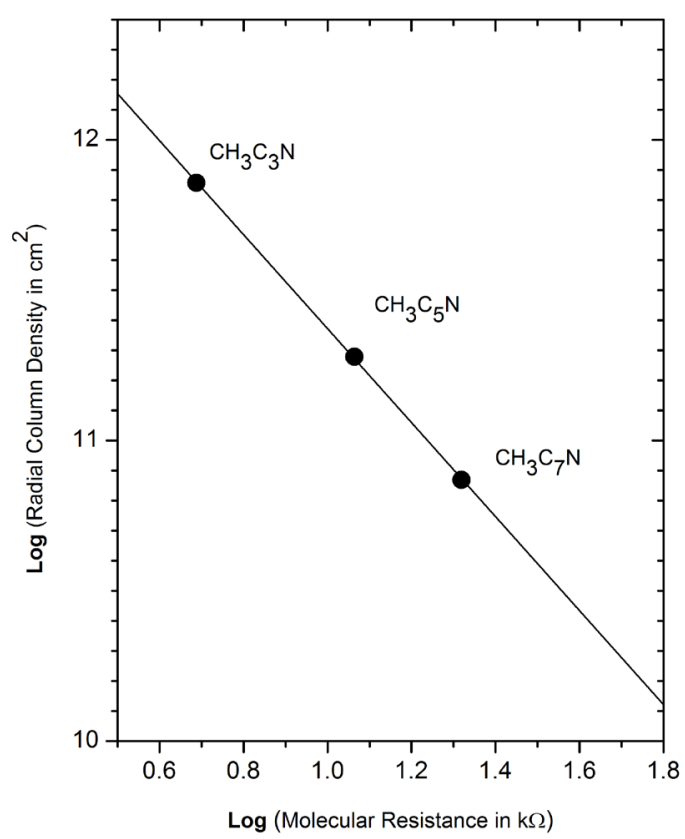

Figure 2. Observed trend between Radial Column Density (RCD) and Molecular Resistance $(R(L))$ in a logarithmic scale.

considered when chemical models are being used in a complex network of reaction schemes under LTE as well as in radial column density estimations of ISM. Effectively, our predictions about how the radial column density of long molecular wires declines with increasing molecular resistance, introduce a new criterion for radio searches of long molecules. The last question is important when a large amount of integration time will be necessary for observing these particular molecular systems.

Other linear molecular wires detected in circumstellar envelopes surrounding carbon-rich stars are being analyzed in our laboratory.

\section{Acknowledgements}

The authors acknowledge to the Centre for Environmental Sciences of the University of Chile for financial support.

\section{References}

[1] Müller, H.S.P., Goicoechea, J.R., Cernicharo, J., Agúndez, M., Pety, J., Cuadrado, S., Gerin, M., Dumas, G. and Chapillon, E. (2014) Revised Spectroscopic Parameters of $\mathrm{SH}^{+}$from ALMA and IRAM $30 \mathrm{~m}$ observations. Astronomy and Astrophysics, 569, L5-L9. http://dx.doi.org/10.1051/0004-6361/201424756

[2] Kahane, C., Ceccarelli, C., Faure, A. and Caux, E. (2013) Detection of Formamide, the Simplest but Crucial Amide, in a Solar-type Protostar. Astrophysical Journal Letters, 763, L38-L42. http://dx.doi.org/10.1088/2041-8205/763/2/L38

[3] Jorgensen, J.K., Favre, C., Bisschop, S.E., Bourke, T.L., van Dishoeck, E.F. and Schmalzl, M. (2012) Detection of the Simplest Sugar, Glycolaldehyde, in a Solar-type Protostar with ALMA. Astrophysical Journal Letters, 757, L4-L9. http://dx.doi.org/10.1088/2041-8205/757/1/L4

[4] Herbst, E. and van Dishoeck, E.F. (2009) Complex Organic Interstellar Molecules. Annual Review of Astronomy and Astrophysics, 47, 427-480. http://dx.doi.org/10.1146/annurev-astro-082708-101654

[5] Pardo, J.R., Cernicharo, J. and Goicoechea, J.R. (2005) Observational Evidence of the Formation of Cyanopolyynes in CRL 618 through the Polymerization of HCN. Astrophysical Journal, 628, 275-282. http://dx.doi.org/10.1086/430774

[6] Woods, P.M., Millar, T.J., Herbst, E. and Zijlstra, A.A. (2003) The Chemistry of Protoplanetary Nebulae. Astronomy and Astrophysics, 402, 189-199. http://dx.doi.org/10.1051/0004-6361:20030215

[7] Millar, T.J., Herbst, E. and Bettens, R.P.A. (2000) Large Molecules in the Envelope Surrounding IRC+10²16. Monthly Notices of theRoyal Astronomical Society, 316, 195-203. 
[8] Cordiner, M.A. and Millar, T.J. (2009) Density-Enhanced Gas and Dust Shells in a New Chemical Model for IRC+10216. Astrophysical Journal, 697, 68-78. http://dx.doi.org/10.1088/0004-637X/697/1/68

[9] Morales, R.G.E. and González-Rojas, C. (2005) Dipole Moments of Polyenic Oligomeric Systems. Part II. Molecular Organic Wire Resistivities: Polyacetylenes, Allenes and Polylines. Journal of Physical Organic Chemistry, 18, 941944. http://dx.doi.org/10.1002/poc.931

[10] McCarthy, M.C., Chen, W., Travers, M.J. and Thaddeus, P. (2000) Microwave Spectra of 11 Polyyne Carbon Chains. Astrophysical Journal, 129, 611-623. http://dx.doi.org/10.1086/313428

[11] Bell, M.B., Feldman, P.A., Kwok, S. and Matthews, H.E. (1982) Detection of $\mathrm{HC}_{11} \mathrm{~N}$ in $\mathrm{IRC}^{2} 10^{\circ} 216$. Nature, 295, 389-391. http://dx.doi.org/10.1038/295389a0

[12] Bell, M.B., Feldman, P.A., Travers, M.J., McCarthy, M.C., Gottlieband, C.A. and Thaddeus, P. (1997) Detection of HC11N in the Cold Dust Cloud TMC-1. Astrophysical Journal Letters, 483, L61-L64. http://dx.doi.org/10.1086/310732

[13] Kroto, H.W., Kirby, C., Walton, R.M., Avery, L.W., Broten, N.W., MacLeod, J.M. and Oka, T. (1978) The Detection of Cyanohexatriyne, $\mathrm{H}\left(\mathrm{CC}_{3}\right) \mathrm{CN}$, in Heiles' Cloud 2. Astrophysical Journal Letters, 219, L133-L137. http://dx.doi.org/10.1086/182623

[14] Smith, D. (1992) The Ion Chemistry of Interstellar Clouds. Chemical Reviews, 92, 1473-1485. http://dx.doi.org/10.1021/cr00015a001

[15] Carter, F.L. (1986) Molecular Electronic Devices. Marcel Dekker Inc., New York.

[16] Joachim, C. and Roth, S. (1997) Atomic and Molecular Wires. Kluwer Academic Publishers, Alphen aan den Rijn. http://dx.doi.org/10.1007/978-94-011-5882-4

[17] James, D.K. and Tour, J.M. (2005) Molecular Wires. Topics in Current Chemistry, 257, 33-62. http://dx.doi.org/10.1007/b136066

[18] Hernández, C. and Morales, R.G.E. (1993) Bridge Effect in Charge-Transfer Photoconduction Channels. 1. Aromatic Carbonyl Compounds. Journal of Physical Chemistry, 97, 11649-11651. http://dx.doi.org/10.1021/j100147a016

[19] Morales, R.G.E. and Hernández, C. (2012) Cyanopolyynes as Organic Molecular Wires in the Interstellar Medium. International Journal of Astronomy and Astrophysics, 2, 230-235. http://dx.doi.org/10.4236/ijaa.2012.24030

[20] Morales, R.G.E. and González-Rojas, C. (1998) Dipole Moments of Polyenic Oligomeric Systems. Part I. A One-Dimensional Molecular Wire Model. Journal of Physical Organic Chemistry, 11, 853-856. http://dx.doi.org/10.1002/(SICI)1099-1395(199812)11:12<853::AID-POC74>3.0.CO;2-Y

[21] González, C. and Morales, R.G.E. (1999) Molecular Resistivities in Organic Polyenic Wires. I. A One-Dimensional Photoconduction Charge Transfer Model. Chemical Physics, 250, 279-284. http://dx.doi.org/10.1016/S0301-0104(99)00335-3

[22] Frisch, M.J., Trucks, G.W., Schlegel, H.B., Scuseria, G.E., Robb, M.A., Cheeseman, J.R., Scalmani, G., Barone, V., Mennucci, B., Petersson, G.A., Nakatsuji, H., Caricato, M., Li, X., Hratchian, H.P., Izmaylov, A.F., Bloino, J., Zheng, G., Sonnenberg, J.L., Hada, M., Ehara, M., Toyota, K., Fukuda, R., Hasegawa, J., Ishida, M., Nakajima, T., Honda, Y., Kitao, O., Nakai, H., Vreven, T., Montgomery Jr., J.A. Peralta, J.E., Ogliaro, F., Bearpark, M., Heyd, J.J., Brothers, E., Kudin, K.N., Staroverov, V.N., Kobayashi, R., Normand, J., Raghavachari, K., Rendell, A., Burant, J.C., Iyengar, S.S., Tomasi, J., Cossi, M., Rega, N., Millam, J.M., Klene, M., Knox, J.E., Cross, J.B., Bakken, V., Adamo, C., Jaramillo, J., Gomperts, R., Stratmann, R.E., Yazyev, O., Austin, A.J., Cammi, R., Pomelli, C., Ochterski, J.W., Martin, R.L., Morokuma, K., Zakrzewski, V.G., Voth, G.A., Salvador, P., Dannenberg, J.J., Dapprich, S., Daniels, A.D., Farkas, O., Foresman, J.B., Ortiz, J.V., Cioslowski, J. and Fox, D.J. (2009) Gaussian 09, Revision A.01. Gaussian, Inc., Wallingford. 